\title{
LOGICS WITH GIVEN CENTERS AND STATE SPACES
}

\author{
PAVEL PTÁK
}

\begin{abstract}
Let $B$ be a Boolean algebra and let $K$ be a compact convex subset of a locally convex topological linear space. Then there exists a logic with the center Boolean isomorphic to $B$ and with the state space affinely homeomorphic to $K$.
\end{abstract}

Introduction. In the quantum logic approach to the foundations of quantum mechanics, one identifies the event structure of a system with an orthomodular partially ordered set $L$ (called usually a logic). The set of states is then represented by the set $\delta(L)$ of all probability measures on $L$ (see $[4,7])$. It can be shown that $\delta(L)$ is a compact convex set and conversely, it was proved by F. W. Shultz [6] that any compact convex subset of a locally convex topological linear space is affinely homeomorphic to $\mathcal{S}(L)$ for a logic $L$.

The center $C(L)$ of a logic $L$ is the subset of $L$ consisting of all "absolutely compatible" elements. It is known that the center of $L$ is a Boolean algebra (see $[1,4])$. Obviously, any Boolean algebra is the center of a logic.

Let us now consider the center and the state space simultaneously. The question is if for any Boolean algebra $B$ and any compact convex subset of a LCTLS there exists a logic $L$ such that $C(L)=B$ and $\delta(L)=K$. We answer the question in the affirmative. In the construction we use, among other tools, the result of Shultz [6] and the technique of $\mathbf{R}$. Greechie [2] for constructing orthomodular posets.

Notions. Results. Let us first review the basic definitions and state some auxiliary propositions.

Definition 1. A logic is a set $L$ endowed with a partial ordering $\leqslant$ and a unary operation ' such that:

(i) $0,1 \in L$;

(ii) $a \leqslant b \Rightarrow b^{\prime} \leqslant a^{\prime}$ for any $a, b \in L$;

(iii) $\left(a^{\prime}\right)^{\prime}=a$ for any $a \in L$;

(iv) $a \vee a^{\prime}=1$ for any $a \in L$;

(v) $\bigvee_{i=1}^{n} a_{n}$ exists in $L$ whenever $a_{n} \in L, a_{n} \leqslant a_{k}^{\prime}$ for $n \neq k$;

(vi) $b=a \vee\left(b \wedge a^{\prime}\right)$ whenever $a, b \in L, a \leqslant b$.

In the sequel, we shall reserve the symbol $L$ for logics. One can prove easily that if $a, b \in L, a \leqslant b^{\prime}$ then $a \vee b, a \wedge b$ exists in $L$.

Definition 2. Two elements $a, b \in L$ are called compatible if there are three elements $c, d, e \in L$ such that $c \leqslant d^{\prime}, d \leqslant e^{\prime}, e \leqslant c^{\prime}$ and $a=c \vee d, b=c \vee e$.

Received by the editors May 27, 1982.

1980 Mathematics Subject Classification. Primary 81B10, 06C15; Secondary 81C20.

(C)1983 American Mathematical Society 0002-9939/82/0000-1022/\$02.00 
Definition 3. An element $a \in L$ is called central if $a$ is compatible to any element of $L$. We denote by $C(L)$ the set of all central elements of $L$ and call $C(L)$ the center of $L$.

Proposition 1. The set $C(L)$ with the operations ' $, \vee, \wedge$ inherited from $L$ is a Boolean algebra.

Proof. See $[1,4]$.

Definition 4. Let $\left\{L_{\alpha} \mid \alpha \in I\right\}$ be a collection of logics. Denote by $\Pi_{\alpha \in I} L_{\alpha}$ the ordinary Cartesian product of the sets $L_{\alpha}$ and endow the set $\Pi_{\alpha \in I} L_{\alpha}$ with the relation $\leqslant$ and the unary operation ' as follows. If $k=\left\{k_{\alpha} \mid \alpha \in I\right\} \in \prod_{\alpha \in I} L_{\alpha}$ and $h=\left\{h_{\alpha} \mid \alpha \in I\right\} \in \prod_{\alpha \in I} L_{\alpha}$, then $k \leqslant h$ (resp. $k^{\prime}=h$ ) if and only if $k_{\alpha} \leqslant h_{\alpha}$ (resp. $k_{\alpha}^{\prime}=h_{\alpha}$ ) for any $\alpha \in I$. The set $\prod_{\alpha \in I} L_{\alpha}$ with the above defined $\leqslant$, ' is called the product of the collection $\left\{L_{\alpha} \mid \alpha \in I\right\}$.

Proposition 2. Let $\left\{L_{\alpha} \mid \alpha \in I\right\}$ be a collection of logics. Then $\Pi_{\alpha \in I} L_{\alpha}$ is a logic. If $C\left(L_{\alpha}\right)=\{0,1\}$ for any $\alpha \in I$ then $C\left(\Pi_{\alpha \in I} L_{\alpha}\right)$ is Boolean isomorphic to the Boolean algebra of all subsets of $I$.

Proof. See [3, 5].

Definition 5. A state on a logic $L$ is a mapping $s: L \rightarrow\langle 0,1\rangle$ such that:

(i) $s(1)=1$;

(ii) if $a, b \in L, a \leqslant b^{\prime}$ then $s(a \vee b)=s(a)+s(b)$.

Let us denote by $\delta(L)$ the set of all states on $L$. By a result of F. W. Shultz [6], any compact convex subset of a LCTLS equals, up to an affine homeomorphism, $\delta(L)$ for a logic $L$ (and vice versa, which is obvious).

Definition 6. A logic $L$ is called poor (resp. rigid) if $\delta(L)=\varnothing(\operatorname{resp} .|\delta(L)|=1)$. It is known (see $[2,6]$ ) that there are (finite) examples of poor and rigid logics.

Proposition 3. Suppose that $L$ is a poor logic. Put $L_{\alpha}=L$ for any $\alpha \in I$. Then $\Pi_{\alpha \in I} L_{\alpha}$ is also a poor logic.

Proof. Take the mapping $f: L \rightarrow \prod_{\alpha \in I} L_{\alpha}$ such that $f(k)=(k, k, k \ldots)$ for any $k \in L$. If $s \in \mathcal{S}\left(\Pi_{\alpha \in I} L_{\alpha}\right)$ then $s f \in \mathcal{S}(L)$.

Definition 7. A mapping $f: L_{1} \rightarrow L_{2}$ is called an embedding if $f$ is injective and the following requirements are satisfied.

(i) $f(1)=1$;

(ii) $f\left(a^{\prime}\right)=f(a)^{\prime}$ for any $a \in L_{1}$;

(iii) $a \leqslant b$ if and only if $f(a) \leqslant f(b)$;

(iv) if $a \leqslant b^{\prime}$ then $f(a \vee b)=f(a) \vee f(b)$.

Proposition 4. Let $K$ be a compact convex subspace of a LCTLS. Take the logic $L_{1}$ constructed in [6, Theorem, $p$. 321]. Thus $\delta\left(L_{1}\right)=K$ and moreover, $C\left(L_{1}\right)=\{0,1\}$ and $L_{1}$ can be embedded into a poor logic $L_{2}$ with $C\left(L_{2}\right)=\{0,1\}$.

PROof. We must assume here that the reader is well acquainted with the paper [6] and with the Greechie representation of logics (see [2]). It follows immediately from 
the construction of [6] that $C\left(L_{1}\right)=\{0,1\}$ (see e.g. the plan of the construction, p. 321). Further, let us consider the Greechie diagram $D_{1}$ of $L_{1}$ and the Greechie diagram $D$ of a finite poor logic $L$ exhibited in [2]. Let us choose "points" $d_{1} \in D_{1}$, $d_{2} \in D$ such that $d_{1}, d_{2}$ belong to exactly one Boolean block of $D_{1}, D$. Form a new Greechie diagram $D_{2}$ by taking the union $D_{1} \cup D$ and then "identifying" the points $d_{1}, d_{2}$. The diagram $D_{2}$ then represents the required logic $L_{2}$.

We are now ready to prove our result.

THEOREM. Let $B$ be a Boolean algebra and let $K$ be a compact convex subset of $a$ LCTLS. Then there exists a logic $L$ such that $C(L)$ is Boolean isomorphic to $B$ and $\delta(L)$ is affinely homeomorphic to $K$.

Proof. We may suppose that $B$ is a Boolean algebra of subsets of a set $A$. Take a logic $M$ such that $C(M)=\{0,1\}, \delta(M)=K$ and denote by $P$ the poor extension of $M$ (Proposition 4). Take a point $a \in A$ and write $L_{c}=P$ if $c \in A-\{a\}, L_{a}=M$. Consider the logic $R=\prod_{d \in A} L_{d}$. The desired logic $L$ will now be obtained as a sublogic of $R$. Let us describe the elements of $L$. An element $r \in R$ belongs to $L$ if and only if there exists a finite partition $\mathscr{P}$ of $A, \mathscr{P}=\left\{A_{i} \mid i=1,2, \ldots, n\right\}$ such that $A_{i} \in B$ for any $i, 1 \leqslant i \leqslant n$, and $r_{p}=r_{q}$ as soon as $\{p, q\} \subset A_{i}$ for an index $i, 1 \leqslant i \leqslant n$. We are to show that $L$ is a logic with $C(L)=B$ and $\delta(L)=K$.

Obviously, $1 \in L$ and if $k \in L$ then $k^{\prime} \in L$. If $k, h \in L, k \geqslant h$ then $k=h \vee(k$ $\left.\wedge h^{\prime}\right)$. Indeed, if $\mathscr{P}, \mathcal{R}$ are partitions corresponding to $k, h$ then $\mathscr{P} \cap \mathcal{R}$ is the partition corresponding to $k^{\prime} \wedge h$. The rest is obvious. Thus $L$ is a logic.

Further, since $C\left(L_{d}\right)=\{0,1\}$ for any $d \in A$ then any central element of $L$ must have only the elements 0,1 for the coordinates. One can check easily that $k=\left\{k_{d} \mid d\right.$ $\in A\}$, where any $k_{d}$ is either 0 or 1 , belongs to $L$ if and only if $D=\left\{d \mid k_{d}=1\right\} \in B$. Consequently, $C(L)=B$.

It remains to prove that $\delta(L)=K$. Since $\delta(M)=K$, it suffices to show that there is an affine homeomorphism $g: \delta(L) \rightarrow \delta(M)$. Assume that $s \in \delta(L)$. For any $m \in M$, denote by $k^{m}$ the element of $L$ which has $m$ for all its coordinates. Define $g(s)$ such that $g(s)(m)=s\left(k^{m}\right)$. We need to show that $g$ is injective.

Let us suppose that $g\left(s_{1}\right)=g\left(s_{2}\right)$. Take an element $k \in L$ and assume that $\mathscr{P}$ is the partition corresponding to $k$. Let $A_{1}$ be such a set of $\mathscr{P}$ that $a \in A_{1}$. Denote by $h=\left\{h_{d} \mid d \dot{\epsilon} A\right\}$ the element of $L$ with $h_{d}=0$ if $d \in A_{1}, h_{d}=1$ otherwise. It follows from Proposition 3 that $s_{1}(k \wedge h)=s_{2}(k \wedge h)=0$. Since $g\left(s_{1}\right)=g\left(s_{2}\right)$, we see, again applying Proposition 3, that $s_{1}(k)=s_{1}\left(k \wedge h^{\prime}\right)=s_{2}\left(k \wedge h^{\prime}\right)=s_{2}(k)$. Hence the mapping $g: \delta(L) \rightarrow \delta(M)$ is injective and the proof is complete.

Let us state explicitly the following special corollary.

Corollary. Given a Boolean algebra B, there exists a poor (resp. rigid) logic $L$ such that $C(L)=B$.

Let us observe in conclusion that a similar method yields an analogous result for $\sigma$-complete logics and $\sigma$-additive states. Naturally, the center then cannot be arbitrary since there are Boolean $\sigma$-algebras without any $\sigma$-additive state. 
THEOREM. Let $B$ be a Boolean $\sigma$-algebra of subsets of a set and let $K$ be a compact convex subset of a LCTLS. Then there is a $\sigma$-complete logic $L$ such that $C(L)$ is Boolean $\sigma$-isomorphic to $B$ and the space of $\sigma$-additive states on $L$ is affinely homeomorphic to $K$.

\section{REFERENCES}

1. J. Brabec and P. Pták, On compatibility in quantum logics, Found. Phys. 12 (1982), 207-212.

2. R. Greechie, Orthomodular lattices admitting no states, J. Combin. Theory Ser. A 10 (1971), 119-132.

3. S. Gudder, Uniqueness and existence properties of bounded observables, Pacific J. Math. 19 (1966), $81-93$.

4. Stochastic methods in quantum mechanics, Elsevier, North-Holland, Amsterdam, 1979.

5. V. Maňasová and P. Pták, On states on the product of logics, Internat. J. Theoret. Phys. 20 (1981), 451-456.

6. F. W. Shultz, A characterization of state spaces of orthomodular lattices, J. Combin. Theory Ser. A 17 (1974), 317-328.

7. V. Varadarajan, Geometry of quantum theory, vol. 1, Van Nostrand Reinhold, Princeton, N. J., 1968.

Department of Mathematics, Technical University of Prague, 16627-Prague 6, CzechosloVAKIA 